\title{
STUDY OF CYTOGENETIC ABNORMALITIES IN G-CSF STIMULATED PERIPHERAL BLOOD CELLS AND NON-STIMULATED BONE MARROW CELLS OF PATIENTS WITH MYELOFIBROSIS
}

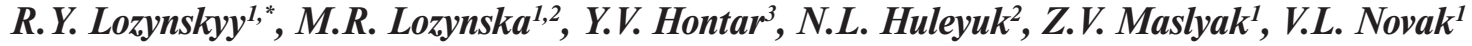 \\ ${ }^{I}$ State Institution "Institute of Blood Pathology and Transfusion Medicine of National Academy of Medical \\ Sciences of Ukraine”, Lviv 79044, Ukraine \\ ${ }^{2}$ State Institution "Institute of Hereditary Pathology of National Academy of Medical Sciences \\ of Ukraine”, Lviv 79000, Ukraine \\ ${ }^{3}$ Medical Center “IGR”, Kyiv 03115, Ukraine
}

The aim of the study was to improve cytogenetic diagnostics and monitoring of myelofibrosis and to reveal the spectrum of cytogenetic abnormalities in patients from Ukraine. Materials and Methods: A total of 42 patients (23 females and 19 males) with myelofibrosis was studied using different cytogenetic methods. Granulocyte colony-stimulating factor (G-CSF) was added by the new method during cultivation of peripheral blood (PB) cells from 31 patients for specific stimulation of mitotic divisions. Two patients underwent examination by fluorescent in situ hybridization method. Results: In cell cultures of PB stimulated in vitro with G-CSF and in non-stimulated bone marrow chromosome abnormalities were found in $19(45.2 \%)$ of all the patients. The spectrum of cytogenetic abnormalities of bone marrow and PB was the same in all of the patients. Aspiration of bone marrow was unsuccessful due to significant fibrosis in $10(29.4 \%)$ of 34 patients. The study by fluorescent in situ hybridization method confirmed cytogenetic abnormalities revealed by G-method and discovered additional possibly normal subclone. Conclusions: Cytogenetic study of PB using in vitro G-CSF as a specific stimulant of mitosis instead of phytohemagglutinin revealed significant variety of chromosomal abnormalities in Ukrainian patients with myelofibrosis. This method could be a less invasive alternative to cytogenetic examination of bone marrow in the subgroup of patients with considerable fibrosis and consecutive changes. The usage of fluorescent in situ hybridization method supplemented karyotyping by G-banding method.

Key Words: myelofibrosis, karyotype, peripheral blood, granulocyte colony-stimulating factor, G-banding, fluorescent in situ hybridization.

Myelofibrosis (MF) as a myeloproliferative neoplasm (MPN) could be either idiopathic (primary) or arise due to transformation of such MPNs as polycythemia vera and essential thrombocythemia. Cytogenetic studies are often crucial for diagnosis of MF according to modern WHO criteria [1], because this requires myeloproliferative changes to be confirmed by clonality markers. Molecular genetic studies give additional opportunities for such confirmation, but they require the usage of expensive and complicated sequencing methods in large amount of the patients. Cytogenetic study of metaphase chromosomes using G-method of differential staining could allow revealing the spectrum of cytogenetic abnormalities of all chromosomes of the cell, including rare changes appearing in the small subclones of pathological cells. Searching for such rare abnormalities is particularly challenging at low mitotic activity of pathological cells and with insufficient quantity of substrate for analysis that often accompanies MF [2]. Appearance of cytogenetic abnormalities and their accumulation could change patient's prognostic risk according

Submitted: December 08, 2015.

${ }^{\star}$ Correspondence: E-mail: roslozynskyy@gmail.com Abbreviations used: BM - bone marrow; DIPSS - dynamic international prognostic scoring system; G-CSF - granulocyte colony-stimulating factor; FISH - fluorescent in situ hybridization; MF - myelofibrosis; MPN - myeloproliferative neoplasm; PB peripheral blood; PHA - phytohemagglutinin. to dynamic international prognostic scoring system (DIPSS) and require to adjust treatment regimen [3]. Currently, the "golden standard" in MF diagnostics is cytogenetic investigation of bone marrow (BM). However, aspiration of BM is often unsuccessful in MF, because of considerable fibrotic changes and replacement of hemopoietic cell clusters onto reticulin and collagen fibers. In this case, repeated punctures of the BM are needed. These manipulations are quite uncomfortable for patients, and even sometimes after several repeats may be ineffective [4]. In the $80^{\text {th }}$ the cytogenetic analysis of peripheral blood (PB) instead of BM was proposed [5]. However, standard methods of $\mathrm{PB}$ examination using phytohemagglutinin (PHA) lead to stimulation of lymphoid cells that usually are not affected in MF. On the other hand, cytogenetic studies of blood without stimulant in most cases cannot provide sufficient mitotic activity to conduct the analysis of metaphase chromosomes in the sample [6]. Fluorescent in situ hybridization (FISH) method can be applied for the detection of cytogenetic abnormalities in MF. The method has higher sensitivity to certain abnormalities of chromosomes than routine karyotyping with G-method. Effectiveness of FISH studies is shown for the diagnostics of myelodysplastic syndromes [7]. However, this high-precision method cannot unveil the whole spectrum of changes in cells' karyotype without the usage of very expensive multiplex FISH approach [8]. An important feature of FISH method is the ability to detect abnormalities in interphase cells, 
unlike the G-method of differential staining that reveals abnormalities in mitotically active cells.

Thus, the methods of cytogenetic analysis in MF should be improved in sensitivity to small pathological cell subclones, as well as become more suitable for larger amount of patients, and should require less painful manipulation at diagnosis and during monitoring of the disease.

The aim of the study was to improve cytogenetic diagnostics and monitoring of MF and to reveal spectrum of cytogenetic abnormalities in Ukrainian patients.

\section{MATERIALS AND METHODS}

A total of 42 patients (23 females and 19 males) with MF were studied. The average age of the patients in the study was about 57 years ( 56.3 years in males and 57.6 in females). 38 patients suffered from idiopathic (primary) MF and in 4 patients secondary MF was diagnosed ( 3 patients had transformation of polycythemia vera to MF, and in 1 patient MF evolved from essential thrombocythemia). Patients were residents of 8 regions of Ukraine: Lviv, Ternopil, Ivano-Frankivsk, Khmelnitsky, Volyn, Vinnytsia, Rivne and Zakarpattya.

Patients were examined according to the protocol of medical care approved by the Ministry of Health of Ukraine. For the study the following laboratory methods were used: complete blood count with cytological examination of blood smears stained with MayGrünwald - Giemsa staining; iliac trephine biopsy with subsequent histological examination of biopsy samples. If the diagnosis was doubtful, immunohistochemical study was performed.

Cytogenetic methods were used. BM aspirate in vitro cultivation followed by subsequent cytogenetic analysis were performed for 25 patients ( 9 males and 16 females). BM samples were prepared by standard method [9] without stimulation. Cytogenetic analysis of PB was performed for 31 patients ( 15 males and 16 females) with immature myeloid progenitor cells in blood smears. Three different methods were used for cultivation of PB. In order to specifically stimulate mitotic division of circulating stem cells, blast cells and other immature myeloid precursors of $\mathrm{PB}$ the granulocyte colonystimulating factor (G-CSF) filgrastim (Dr. Reddy's, India) was added during cell cultivation for the cytogenetic blood samples of all 31 patients (patent of Ukraine application № a201505498 from 04.06.2015). 10 patients ( 5 males and 5 females) among these 31 were selected for a control group. PB for this group was also cultivated with 2 other methods: without any stimulation and with addition of PHA (Gibco, USA) [10]. In 12 patients (5 males and 7 females) cytogenetic study was performed for both PB and BM. Also 10 patients underwent cytogenetic reexamination of the PB with G-CSF stimulation from 6 to 12 months after the initial examination.

In all cases in vitro 24 hours cultivation with RPMI 1640 culture medium and fetal calf serum was performed. To stop mitosis at metaphase colchicine solution was used. Cells were fixed by standard methods. G-Method of differential staining of metaphase chromosomes was used. Cytogenetic analysis was performed with light microscope at magnification $10 \times 100$ using recommendations of ISCN [11, 12].

Before the collection of the samples for cytogenetical study the patients were recommended to stop any cytotoxic therapy, i.e. hydroxyurea or interferonalpha for the period of 3 to 7 days in order to maintain cell proliferation and to avoid distortion of the results of cytogenetic analysis.

To confirm and clarify cytogenetic abnormalities in 2 patients additional studies of interphase PB cells by FISH method were carried out after using G-banding method of differential staining. For one of them the study by FISH method was repeated after 12 months, and for another patient also interphase nuclei of cells in BM were examined. The mixture of FISH probes for centromeric regions of chromosome 3 (D3Z1) and 9 (D9Z1) were used: CEP3 (Spectrum Orange), CEP9 (Spectrum Aqua), (Abbott, USA). For centromeric regions and long arms of chromosome 8 (D8Z2 and TRPS1) the mixture of FISH probes CEP8 (Spectrum Aqua) and LSI8q23.3 (Spectrum Red) (Cytosell, UK). For subsequent loci of chromosome 5, such as 5p15.2 (CTNND2), 5p15.31 (FLJ25076) and $5 q 35$ (SOTOS) - a mixture of probes LSI5p15.2 (Spectrum Red), LSI5p15.31 (Spectrum Green), LSI5q35 (Spectrum Green) (Cytosell, UK) were used.

The study received permission of the local ethics committee and was conducted according to the principles of the Helsinki Declaration of Human Rights, the European Convention on Human Rights and Biomedicine, and the laws of Ukraine. All the patients subscribed informed consent for the participation in the study. However, 8 patients refused to undergo BM puncture, so, the cytogenetic investigation of BM wasn't performed for these 8 patients.

\section{RESULTS AND DISCUSSION}

Cytogenetic analysis of PB samples without stimulation of cell division was unsuccessful in all 10 patients of control group due to either insufficient quantity and quality of metaphase plates or lack of mitosis in the samples. If PHA was used to stimulate lymphocytes in venous blood, normal karyotype was revealed in every patient of this control group, because lymphoid cells in MF were theoretically unaffected by the disease, as expected. However, in cell cultures of PB leukocytes stimulated in vitro with G-CSF and in non-stimulated BM samples chromosome abnormalities were detected in $19(45.2 \%)$ of all the patients. In $23(54.8 \%)$ of patients normal karyotype was found. The results of the cytogenetic study of the cells obtained from G-CSF-stimulated PB and non-stimulated $\mathrm{BM}$ in patients with MF are presented in the Table.

Chromosome abnormalities were detected in 9 male patients and 10 females. Among patients with cytogenetic abnormalities only one clonal abnormality of karyotype was revealed in 10 patients (52.6\%). Two chromosomal abnormalities were observed in 3 patients (15.8\%). 6 patients $(31.6 \%)$ with cytogenetic abnormalities had initially complex karyotype. Abnormalities 
of chromosome 9 were the most common, and were observed in 6 patients (31.6\% of patients with karyotype changes), and in 4 of them monosomy of chromosome 9 was detected.

Table. Spectrum of cytogenetic abnormalities in patients with MF

\begin{tabular}{|c|c|c|c|}
\hline No. & $\begin{array}{c}\text { Gen- } \\
\text { der/Age } \\
\text { (years) }\end{array}$ & $\begin{array}{l}\text { Ma- } \\
\text { teri- } \\
\text { al }\end{array}$ & Karyotype \\
\hline 1 & $\mathrm{M} / 59$ & BM & $46, \mathrm{XY}[12] / 47, \mathrm{XY},+8[2] / 93, \mathrm{XXYY}[2] / 4 \mathrm{n} \pm[1]$ \\
\hline 2 & $\mathrm{M} / 27$ & PB & $\begin{array}{l}46, \mathrm{XY}[16] / 46, \mathrm{XY}, \text { del5p12[2]/46,XY,del9q21q32[1]/ } \\
46, \mathrm{XY}, \operatorname{der} 15[11 / 4 n \pm[1]\end{array}$ \\
\hline & & BM & $\begin{array}{l}\text { 46,XY[16]/46,XY,del5p12[1]/46,XY,der(15)/ } \\
{[1] / 46, X Y, \operatorname{del} 9 q 21 q 32[1] / 4 n \pm[2]}\end{array}$ \\
\hline 3 & $M / 59$ & BM & $\begin{array}{l}50, \mathrm{XY},+1,+2,+19,+\operatorname{mar} 1[7] / \\
/ 59, \mathrm{XY}, \text { idem },+6,+8,+9,+11,+20,+21,+22,+ \text { mar2, } \\
+ \text { +mar3[2] }\end{array}$ \\
\hline 4 & $F / 41$ & $\begin{array}{l}\text { PB } \\
\text { BM }\end{array}$ & $\begin{array}{l}46, \mathrm{XX}[13] / 47, \mathrm{XX},+8[3] \\
46, \mathrm{XX}[5] / 47, \mathrm{XX}+8[2]\end{array}$ \\
\hline 5 & $F / 60$ & BM & $46, X X[14] / 47, X X,+12[1] / 3 n \pm[2] / 4 n \pm[4]$ \\
\hline $\begin{array}{l}0 \\
7\end{array}$ & $\begin{array}{l}F / 54 \\
F / 67\end{array}$ & $\begin{array}{l}\mathrm{BM} \\
\mathrm{PB} \\
\mathrm{PB}^{\star} \\
\mathrm{BM}\end{array}$ & $\begin{array}{l}46, X X[15] / 4 n \pm[3] \\
49, X X,+3,+8,+9[16] / 48, X X,+3,+9[1] / 46, X X[1] \\
49, X X,+3,+8,+9[19] / 46, X X[1] \\
49, X X,+3,+8,+9[8]\end{array}$ \\
\hline 8 & $F / 52$ & $\begin{array}{l}\mathrm{PB}^{*} \\
\mathrm{~PB}^{*} \\
\mathrm{BM}\end{array}$ & $\begin{array}{l}46, X X[4] / 44, X X,-5,-17[2] / 45, X X,-17[2] \\
46, X X[1] / 46, X X, i(17 q)[10] \\
46, X X[4] / 43, X X,-5,-17,-18[3] / 44, X X,-5,-17[3] / 45, X X,-17[4]\end{array}$ \\
\hline 9 & $\mathrm{M} / 54$ & $\begin{array}{l}\mathrm{PB}^{2} \\
\mathrm{~PB}^{\star}\end{array}$ & $\begin{array}{l}46, X Y, 14 \mathrm{ph}+[5] / 45, X Y, 14 \mathrm{ph}+,-7[3] \\
46, X Y, 14 \mathrm{ph}+[10] / 44, X Y, 14 \mathrm{ph}+-7,-9[2] / 4 \mathrm{n} \pm[1]\end{array}$ \\
\hline 10 & $F / 65$ & $\mathrm{~PB}$ & 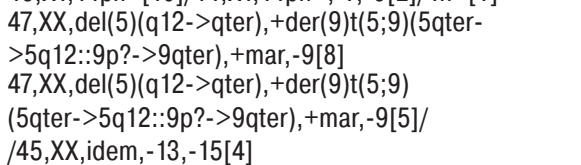 \\
\hline 11 & $M / 69$ & PB & $\begin{array}{l}44, X Y,+ \text { rea(1),+8,+mar, }-3,-5,-7,-15,-17[5] / \\
/ 39, X Y, \text { idem, }-2,-6,-11,-19,-21[1]\end{array}$ \\
\hline 12 & $F / 72$ & $\begin{array}{l}\mathrm{PB} \\
\mathrm{PB}^{*}\end{array}$ & $\begin{array}{l}46, X X[14] 4 n \pm[2] \\
46, X X[18] / 45, X X,-22[3]\end{array}$ \\
\hline 13 & $M / 60$ & PB & $46, X Y[11] / 47, X Y,+r e a(1)[2]$ \\
\hline 14 & $M / 34$ & PB & $46, X Y[11] / 44, X Y,-15,-Y[2] / 45, X Y,-Y[1]$ \\
\hline 15 & $M / 62$ & PB & $46, X Y[12] / 45, X Y,-3[2] / 43, X Y, i d e m,-9,-21[1]$ \\
\hline 16 & $\mathrm{~F} / 48$ & $\begin{array}{l}\text { PB } \\
\text { BM }\end{array}$ & $\begin{array}{l}46, X X[5] / 45, X X,-7[3] \\
46, X Y[1] / 45, X Y,-7[3]\end{array}$ \\
\hline 17 & $F / 59$ & $\mathrm{~PB}$ & $46, X X[10] / 46, X X, 20 \mathrm{q}-[3] / 44, X X$, idem, - $17,-22[2]$ \\
\hline 18 & $\mathrm{~F} / 69$ & $\begin{array}{l}\text { PB } \\
\text { BM }\end{array}$ & $\begin{array}{l}47, X X,+2,+13,-7[5] / 45, X X,+ \text { idem },-7,-8,-21[1] \\
47, X X+2+13,-7[3]\end{array}$ \\
\hline 19 & $M / 35$ & $\begin{array}{l}\mathrm{PB} \\
\mathrm{BM}\end{array}$ & $\begin{array}{l}46, X Y[6] / 45, X Y,-7[3] \\
46, X Y[1] / 45, X Y,-7[3]\end{array}$ \\
\hline
\end{tabular}

Note: $\mathrm{M}$ - male; $\mathrm{F}$ - female; $\mathrm{PB}$ - peripheral blood; BM - bone marrow; $\mathrm{PB}^{\star}$ - cytogenetic reexamination of peripheral blood.

Trisomies were diagnosed in 7 patients (36.8\%). Among frequently detected trisomies there were additional copies of chromosomes $1-3,6,8,9,11-13$ and 19. Trisomy of chromosome 8 was the most commonly revealed, being found in 4 patients. Among other abnormalities frequently noted in the literature, the deletion $20 q 11$ was identified in only one patient as a small subclone. According to molecular genetic studies this abnormality could be present in lower percentage of PB cells comparing to BM cells [13]. BM collection for cytogenetic study was not always possible in our study, and PB studies were performed instead. This could be an explanation of the rarity of this abnormality. Falsenegative results could also appear due to difficulties in detection of small deletions of 20q using G-method.

In patients with MF structural rearrangements were presented by deletions, inversions and translocations involving long and short arms of chromosomes 1, 5, 9, 17, and 20. Complex translocations were found in $6 \mathrm{pa}-$ tients, and 2 of them had 2 or more additional abnormal chromosomes appeared as a result of translocations.
Also, in some patients fractures in centromere regions of chromosome 9 were revealed.

In 1 patient (sample No. 11 in the Table) with a complexkaryotype presented by additional copies of chromosome 8, chromosome 1 derivatives and other rearrangements, abnormal mitosis with complete fragmentation of chromosomes was revealed. This phenomenon is assumed as a biomarker of mitotic cell death and elimination of genetically unstable cells. It was established that the more severe morphological anaplasia of tumors and the higher mitotic activity of cells is, the more common abnormal mitosis occur [14].

The rearrangements considered as prognostically unfavorable according to IPSS/DIPSS Plus were observed in 9 patients $(47.4 \%$ among patients with abnormalities of chromosomes). Hypodiploid clonal karyotype was revealed in 4 patients. The most frequent were losses of chromosomes 7 and 9, and monosomy of chromosome 5 was slightly more rare. Besides complex karyotype, trisomy of chromosome 8 and monosomy of chromosomes 5 and 7 , other unfavorable cytogenetic abnormalities were revealed, such as del(5)(q12.1-qter) and isochromosome i(17q).

In patients with MF cell clones with increased ploidy to $3 n \pm$ and more frequently $4 n \pm$ (samples No. 2, 5, 6, 12 in the Table) were detected. Polyploid cells were identified in 5 patients, and 4 of them had a combination with other chromosome abnormalities.

Polyploidy was a notable feature of the cells in patients with MF, because it implied copy number increase of mutated genes. Such changes could cause the appearance of clonal benefits through enhanced ability to proliferate and could lead to higher genetic instability through greater chance of chromosomes' abnormal disjunction during mitosis followed by significant quantitative changes of chromosomes.

In all patients with detected chromosomal abnormalities the mosaic karyotype with the number of cell clones from 2 to 4 (mostly both abnormal and normal) was observed. Only in $2(5 \%)$ patients no cellular subclones with normal karyotype were detected. This implied genetic heterogeneity of the hematopoietic cells and the possibility of subclone detection using G-method of cytogenetic analysis in this disease. This also could confirm the coexistence of normal and pathological hematopoiesis in almost all patients with MF.

So, we could speculate about the potential of this disease to be curable in case of effective elimination of abnormal cells during the treatment process without allogenic hemopoietic stem cells transplantation, since high proportion of patients with cytogenetically normal subclones has been revealed.

Aspiration of BM was unsuccessful due to significant fibrosis in 10 (29.4\%) of 34 patients which agreed to undergo BM puncture. In 3 cases (2 cases for the study of BM and 1 case for G-CSF-stimulated PB) karyotyping was unsuccessful due to lack of metaphase plates in the samples (low mitotic rates). So, the results of both $\mathrm{PB}$ and BM simultaneous cytogenetic analysis were accessible in only 12 pa- 
tients. There were no cases where cytogenetic study of BM showed abnormal karyotype along with normal karyotype of G-CSF stimulated PB, and vice versa. The spectrum of cytogenetic abnormalities of $B M$ and PB was the same in all the patients. However, in some patients the quantity of the cells in exact cellular clones was different. In case of such quantity differences in polyploid cell clones a possible explanation is the presence of an increased number of megakaryocyte precursors able to became polyploid in BM.

To reduce the quantity of invasive diagnostic procedures, such as repeated BM punctures, cytogenetic reexamination of the patients was performed using G-CSF stimulated PB from 6 to 12 months after initial examination. $3(30 \%)$ of a total of 10 patients with cytogenetic reexamination performed had new clonal findings in the karyotypes (No. 8, 10, 12 in the Table). Such clonal abnormalities as $\mathrm{i}(17 \mathrm{q})$, monosomies of chromosomes 13, 15, and 22 were revealed in these patients. In one another case, patient's abnormal complex karyotype remained unchanged after 6 months of observation (No. 7 in the Table). The clinical condition of all these 4 patients had been exacerbating with worsening of anemia, splenomegaly and weakness. Two of them died in 6 months after reexamination. In other patients with no clonal chromosomal abnormalities at the initial cytogenetic examination, no new clonal karyotype abnormalities were revealed.

For both 2 patients whom the study with FISH probes was made, the cytogenetic findings revealed by G-method in BM and G-CSF stimulated PB were confirmed. The study by FISH method revealed the same trisomies of chromosomes both in BM and in G-CSF-stimulated PB of the patient No. 7 (see the Table). Below is a brief description of another case with confirmation of the origin of the marker chromosomes using FISH-method.

Clinical example. 65 years old female patient with hepatosplenomegaly, portal hypertension, and severe anemia was diagnosed as idiopathic MF after histological examination of trephine biopsy material and molecular genetic evaluation of JAK2V617F mutation. Extensive reticulin and collagen fibrosis of $\mathrm{BM}$ along with the presence of the mutation in JAK2 gene were confirmed. Leukogram showed significant shift to myelocytes (18\%), metamyelocytes (16\%), and band neutrophils (10\%). High concentration of immature nucleated erythroid precursors (10 erythrokaryocytes to 100 leukocytes) in the PB smear was revealed. The patient underwent periodical treatment with hydroxyurea. Repeated puncture of the sternum and iliac crest to aspirate BM failed, and therefore, it was not possible to conduct cytogenetic studies using standard techniques. The cytogenetic study of PB by culturing cells in vitro using G-CSF was performed instead. The mitotic index of PB cells was high enough to obtain metaphase plates for karyotyping. As a result of the study, the following karyotype was revealed: $47, \mathrm{XX}$, del(5)(q12->qter), $+\operatorname{der}(9) \mathrm{t}(5 ; 9)$ (5qter->5q12::9p?->9qter),+mar,-9[8].
The involvement of short arms of chromosome 9, where JAK2 gene is situated, could possibly lead to additional activation of the mutated gene and give clonal advantage for the pathologically changed cells.

For the analysis of the patient's PB cells' interphase nuclei with FISH method the fluorochrome labels for short and long arms of chromosome 5 (Fig. 1) were used to detect cytogenetic abnormalities quantitatively and to prove the presence of deleted chromosome fragments and derivatives.

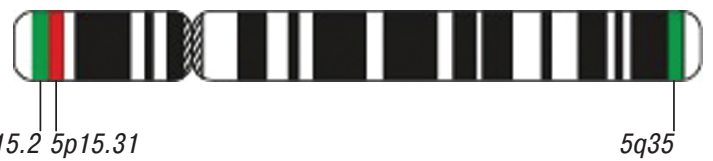

Fig. 1. Schematic view of chromosome 5 with loci 5p15.31 (FLJ25076), 5p15.2 (CTNND2) and 5q35 (SOTOS) labeled by FISH method with a mixture of probes LSI5p15.31 (Spectrum Green), LSI5p15.2 (Spectrum Red) and LSI5q35 (Spectrum Green)

58 cell nuclei from 100 analysed showed 3 signals from short arms and 2 signals from long arms of chromosome 5 (Fig. 2, a). In other 38 cell nuclei normal count of 2 signals for both short and long arms of chromosome 5 were detected (Fig. 2, b). In 4 cells the only one signal from long arm and 2 signals from short arms were revealed (Fig. 2, c). As far as only 2 short and 2 long arms of chromosome 5 had been identified by cytogenetic analysis using G-method, it could be assumed that chromosome 5 was involved to the formation of the marker chromosome in $58 \%$ of the PB cells, where three signals from the genetic material of chromosome's 5 short arms were confirmed by FISH method.

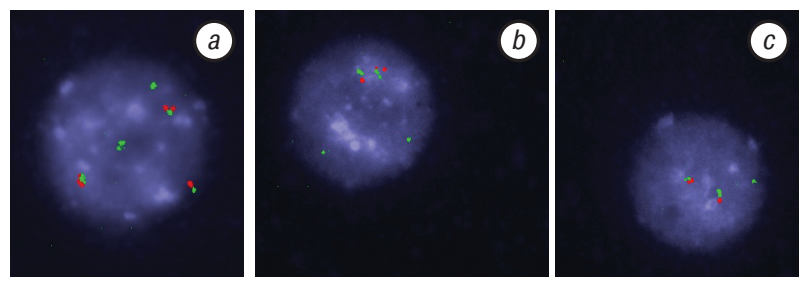

Fig. 2. Nuclei of interphase cells of venous blood of the patient with abnormalities of chromosome 5 labeled by FISH method (magnification 10×100): a) 3 signals from short arms (combined red and green) and 2 signals from long arms (green only) of chromosome 5 (CTNND2x3,FLJ25076x3,SOTOSx2); b) normal count of 2 signals for both short (combined red and green) and long (green only) arms of chromosome 5 (CTNND2,FLJ25076,SOTOS) $\times 2 ; c) 1$ signal from the long arm (green only) and 2 signals from the short arms (combined red and green) of chromosome 5 (CTNND2x2,FLJ25076x2,SOTOSx1)

Cytological analysis of PB smears of the patient at the time of cytogenetic analysis revealed that $77.3 \%$ of leukocytes had myeloid origin, $13.6 \%$ of PB were from lymphoid lineage, and $9.1 \%$ were immature nucleated erythroid precursors. Thus, the number of the cells of non-myeloid origin that theoretically shouldn't be affected in MF was significantly lower than the number of cells with normal quantity of fluorescent signals according to the study by FISH method. This could indicate the presence of a subclone of myeloid cells with normal karyotype undetected by G-banding cytogenetic analysis.

The reexamination after one year of observation to assess the dynamics of cytogenetic changes was 
made also using in vitro G-CSF-stimulated PB. A total of 17 metaphase plates of satisfactory quality were obtained, and the following karyotype was revealed:

$47, X X$, del ( 5 ) ( q $12->$ qter $),+\operatorname{der}(9) \mathrm{t}(5 ; 9)$ ( 5 qter->5q 12::9p ? - >9qter), +mar, - 9 [5]/ /45,XX,idem,-13,-15[4].

Thus, clonal evolution of the cells with the appearance of additional monosomy was detected along with the changes observed in the first examination. Therefore, dynamic cytogenetic study of G-CSF stimulated PB could in this case provide confirmation of disease progression. The presence of all these chromosomal abnormalities could attribute the patient to high risk according to the DIPSS-Plus [3], though without these cytogenetic data, she was assigned to the intermediate-2 risk group. The patient's clinical condition was consistent with high risk group, as predicted by cytogenetics, while being deteriorated during observation. She became dependent from transfusions of red blood cell concentrate. Weakness, spleen and liver volumes progressively increased, 1-2\% of blast cells and promyelocytes appeared in PB leukocyte count in 6 months, despite active therapy. Neither erythropoietins followed by thalidomide + prednisolone could relieve anemia, nor further use of interferon-alpha could influence hepatosplenomegaly and PB changes. Thus, the application of our method of cytogenetic investigation with G-CSF stimulation allowed in this case to obtain more information of potentially important diagnostic and prognostic value. Application of FISH method of PB examination after G-banding allowed to determine anomalies that are difficult to detect using differential staining of metaphase chromosomes. FISH method increased sensitivity of cell subclones detection.

In conclusion, cytogenetic investigation of $\mathrm{PB}$ and $\mathrm{BM}$ in MF revealed a significant variety of chromosomal abnormalities in patients from Ukraine. Cytogenetic studies of PB using in vitro specific stimulant G-CSF instead of PHA in this disease may be a good alternative to cytogenetic examination of BM in the subgroup of patients with prominent fibrosis and consecutive changes. PB studies reduce the need of painful invasive diagnostic manipulations and facilitate followup of the patients.

\section{REFERENCES}

1. Tefferi A, Thiele J, Orazi A, et al. Proposals and rationale for revision of the World Health Organization diagnostic criteria for polycythemia vera, essential thrombocythemia, and primary myelofibrosis: recommendations from an ad hoc international expert panel. Blood 2007; 110: 1092-7.

2. Dos Santos LC, da Costa Ribeiro JC, Silva NP, et al. Cytogenetics, JAK2 and MPL mutations in polycythemia vera, primary myelofibrosis and essential thrombocythemia. Rev Bras Hematol Hemoter 2011; 33: 417-24.

3. Gangat N, Caramazza D, Vaidya R, et al. DIPSS Plus: a refined dynamic international prognostic scoring system for primary myelofibrosis that incorporates prognostic information from karyotype, platelet count, and transfusion status. J Clin Oncol 2011; 4: 392-7.

4. Tefferi A, Meyer RG, Wyatt WA, Dewald GW. Comparison of peripheral blood interphase cytogenetics with bone marrow karyotype analysis in myelofibrosis with myeloid metaplasia. Br J Haematol 2001; 115: 316-9.

5. Pakkala A, Ruutu T, Partanen S, et al. Peripheral blood cells in the study of chromosome aberrations of patients with chronic myeloid leukaemia or myelofibrosis. Acta Haematol 1983; 70: 264-8.

6. Hussein K, Ketterling RP, Hulshizer RL, et al. Peripheral blood cytogenetic studies in hematological neoplasms: predictors of obtaining metaphases for analysis. Eur J Haematol 2008; 80: 318-21.

7. Cherry AM, Slovak ML, Campbell LJ, et al. Will a peripheral blood (PB) sample yield the same diagnostic and prognostic cytogenetic data as the concomitant bone marrow (BM) in myelodysplasia? Leuk Res 2012; 36: 832-40.

8. Anderson R. Multiplex fluorescence in situ hybridization (M-FISH). Methods Mol Biol 2010; 659: 83-97.

9. Czepulkowski BH, Bhatt R, Rooney DE. Basic techniques for the preparation and analysis of chromosomes from bone marrow and leukaemic blood. In: Rooney DE and Czepulkowski BH, eds. Human Cytogenetics. Malignancy and abnormalities: A practical approach. Oxford: Oxford University Press, 1992. 26 p.

10. Hungerford DA. Leukocytes cultured from small inocula of whole blood and the preparation of metaphase chromosomes by treatment with hypotonic KCl. Stain Technol 1965; 40: 333-8.

11. Simons A, Shaffer LG, Hastings RJ. Cytogenetic nomenclature: changes in the ISCN 2013 compared to the 2009 edition. Cytogenet Genome Res 2013; 141: 1-6.

12. Shaffer LG, Slovak ML, Campbell LJ, eds. International system for human cytogenetic nomenclature. Basel: S Karger, 2009. 140 p.

13. Asimakopoulos FA, Holloway TL, Nacheva EP, et al. Detection of chromosome 20q deletions in bone marrow metaphases but not peripheral blood granulocytes in patients with myeloproliferative disorders or myelodysplastic syndromes. Blood 1996; 87: 1561-70.

14. Stevens JB, Liu G, Bremer SW, et al. Mitotic cell death by chromosome fragmentation. Cancer Res 2007; 67: 7686-94. 